\title{
Automated Attendance System Using Facial Recognition
}

\author{
R.Angeline ${ }^{1}$, Kavithvajen. $\mathrm{K}^{2}$, Toshita Balaji ${ }^{3}$, Venugopal Balaji ${ }^{4}$, Malavika Saji ${ }^{5}$ \\ ${ }^{1}$ (CSE, Assistant Professor, SRM Institute of Science and Technology, Chennai) \\ $2,3,4,5$ (CSE, SRM Institute of Science and Technology, Chennai)
}

\section{Abstract:}

The human face is a unique feature and thus it can used in the case of student's attendance. This project is aimed at implementing an automated attendance system. The proposed system framework utilises face detection and recognition techniques. The algorithms implemented are Haar's Cascades and LBPH. The system identifies the correct students and their participation in the class with the help of a camera that records the class and a computer that analyses the feed it receives from the camera. The attendance records are stored in a database maintained by the developers and accessible to the management of the institution.

Keywords - Facial Recognition, Feature Extraction, Training, Linear Binary Patterns Histogram , Biometrics

\section{INTRODUCTION}

Computer Vision is an interdisciplinary field that works on enabling computers to see, identify and process images in the same way that humans do. Main tasks include acquiring, processing, analysing and understanding digital images or videos to produce information. The applications of computer vision are biometrics, object detection, gesture analysis, facial recognition, robotics and many more. These applications can be utilised to improve human lifestyle and ease regular mundane tasks. One such problem is recording and maintaining attendance.

Attendance systems are used to track and monitor when students or employees start and stop work. It enables higher authorities to maintain a record of the number of working hours. The traditional systems have become cumbersome and hard to maintain. In educational institutions and corporates, individuals signing registers and manually counting the hours is just illogical. By practising manual recording in educational institutions, the staff may experience difficulty in both verifying and maintaining each student's record in a classroom environment on a regular basis, especially in classes attended by a large number of students. The manual system also requires more time for recording and calculating the average attendance of each enrolled student.

Humans use their eyes and brain to sense the world around them, so why not use this technique in attendance records and automate the entire process? Biometric verification came into existence and has made these rudimentary systems extinct, as the newer automated ways are usually more efficient and ac- curate. Various human parts are utilised in verifying the identity of individuals like fingerprints, iris scanning and facial features.

Facial recognition is a category of biometric software that maps an individual's facial features mathematically and stores it as a faceprint. This can be used to identify individuals and mark their presence in an attendance system. The face is a unique part of a human body that is nearly impossible to forge and is one of the best ways of biometric verification.

In this project, the technique utilised for user authentication is human face recognition. Facial recognition uses deep learning algorithms to compare live image or video feed to the stored faceprint in order to verify the identity of the individual. The backend of the system calculates the attendance for each individual based on the amount of time his/her face is detected and recognised. This system makes the maintenance of attendance records effortless.

\section{PROPOSED METHOD}

\section{A. Face Detection}

The objective of this module is finding the faces in an image and extracting the features to be used by the face recognition algorithm. Faces of the students present in the class are captured and detected using Haar-cascades. Haar-cascade is an object detection algorithm used to detect faces and facial expressions in an image. In Haar-cascade, the system is provided with various positive images like faces of different people with different backgrounds and negative images which are not faces. The algorithm implements techniques like Ada- 


\section{International Journal of Engineering and Techniques - Volume 4 Issue 2, Mar-Apr 2018}

boost and Integral Images to correctly identify the faces in a single frame.

\section{B. Face Recognition}

Face recognition is responsible for finding characteristics that best describe the face. The Facial Recognition system compares the input facial image with all facial images from a database with the aim to identify the student that matches the face. The detected face is identified using Linear Binary Pattern Histogram algorithm. This algorithm is one of the easiest face recognition algorithms. It represents local features in the images that are compared with the pre-stored dataset. It provides information about the shape and texture of the image describing detailed characteristics that helps in authenticating the process accurately.

\section{Creating a database}

The images of the students that are to be trained, is stored in a database. This module is completed before the live recognition process starts. The user or student holds the main role. The process involved is that the facial recogniser compares the input facial image with the images present in this database to verify the student's identity and presence in the classroom.

\section{Linking the attendance database}

A student will be given attendance once his/her face is identified for an appropriate amount of time. This is stored in a separate database where the corresponding student's name and ID are updated with the attendance. The attendance log will be updated after every period and is accessible only by the admin. This report is then shared with the administers of the Institute.

\section{III.FEATURE EXTRACTION AND RECOGNITION SYSTEM}

\section{A. Haar cascade detection}

Haar Cascade is an effective object detection algorithm. In this algorithm, cascades are trained from the input images to detect a particular object (in our case, a face). The cascades are nothing but features with particular characteristics like edges, colour differences and lines as shown in Figures 1 and 2. The object referred here is a face, hence the cascades look for differences in skin, nose bridge edges and eyes as characteristics. This is then used to detect faces in unknown input images. Initially, the algorithm needs a lot of positive and negative images to train the classifier. Positive images are where the images with the human faces are trained. Negative images are the ones without human faces. It extracts features from the positive images that help to uniquely identify a human face. The value of each feature is obtained by subtracting the pixel value of the white rectangle to the black rectangle. For each calculation, the summation of all pixels of white and black rectangles are to be found. This results in a large amount of data that can slow down the process.

To simplify this, integral images were introduced which made the calculation simpler for a huge amount of data. Even in this, a lot of these cascades are irrelevant with respect to the object to be detected, in this case, the face of the individual. To eradicate training the classifier with irrelevant data, Adaboost was introduced.

This method was introduced to reduce the internal processes. Once the features are identified, it finds the best characteristic that describes the image. Based on this, the faces are categorised as positive and negative. It selects the features with minimum error rates. The final classifier chosen is a sum of these weak classifiers. It is called weak as only one weak classifier cannot determine the features but the summation of weak classifiers define a category of features. With the usage of this method, there is a drastic reduction in the number of selected features. The obtained classifiers are applied to the image for detection. In an image, most of the image region is non-face region. Hence, it is easier to remove images that do not have a face region. For this, Cascade of Classifier was introduced. Instead of applying all the features at one go, this method groups the features into different stages of categories and it is applied one by one. If a window fails the first stage, it is discarded. The algorithm continues the process to drop images that don't satisfy the respective stages and the window that passes all of the stages is the face region.

In the proposed system, to train the classifiers, the pretrained classifier that is available in OpenCV is implemented.

\section{B. Local Binary Patterns Histogram}

LBPH is found to be an efficient technique for texture classification. It uses 4 parameters - Radius, Neighbours, Grid X and Grid Y. The radius is used to build the circular local binary pattern and represents the radius around the central pixel. It is usually set to 1 . The neighbouring sample points are used to build the circular pattern with the radius. It is usually set to 8. Grid X and Grid Y are the number of cells in the horizontal and vertical axis. The algorithm is first trained with the dataset that has the facial images of the people to be recognised. Each image is given an ID so that the algorithm will use this information to recognise the input image. The computational process of the algorithm is as below:
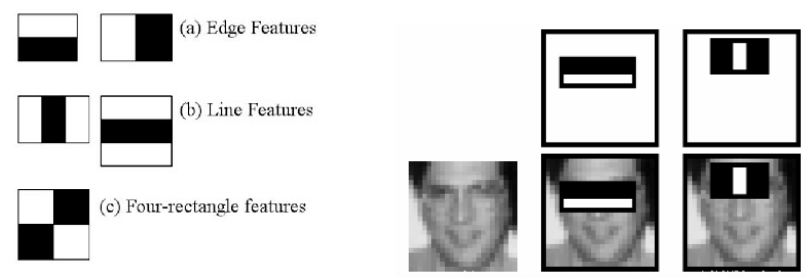

Figure 1: Feature extraction
Figure 2: Cascade of classifiers 
International Journal of Engineering and Techniques - Volume 4 Issue 2, Mar-Apr 2018

I.The unknown image is converted into greyscale.

II.The greyscale image is broken into smaller 3 X 3 matrixes and the central pixel is determined. As shown in Figure 3 , this central value is used to calculate the values of the 8 neighbouring pixels to form the pattern.

III. Each neighbouring pixel is compared to the central pixel. If the greyscale value of the neighbouring pixel is higher than the central pixel, then it is given the digit 1 . Otherwise, it is given the value 0 .

IV. From the previous step, an eight-digit binary value is obtained which is converted into its corresponding decimal value.

V.This decimal value results in a new image with detailed

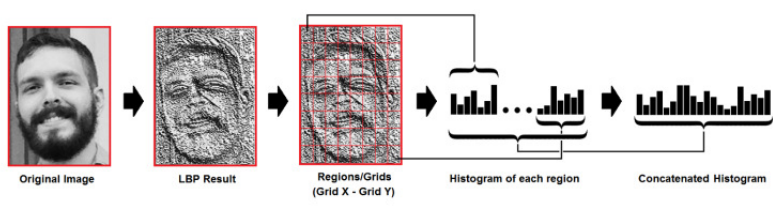

Figure 4: Formation of Histograms in

characteristics.

VI.This resultant image is divided into grids and converted into a histogram as shown in Figure 4. The facial recognition procedure starts after the above training process. The histogram of the input image is compared with the resulted histogram that was computed. The method for this comparison is done by finding Euclidian Distance:

$$
D=\sqrt{\sum_{i=1}^{n}\left(\text { hist } 1_{i}-h i s t 2_{i}\right)^{2}}
$$

The final output of the algorithm is the ID of the image if the comparison was successful. In this system, the existing $\mathrm{LBPH}$ function in the OpenCV library is used.

\section{IV.SYSTEM DESIGN}

The block diagram of the proposed system is presented in Figure 5. The faces of students in the class are acquired during the admission process and is used to create a huge dataset. These images are then trained by the recogniser which produces a trainer file with the extension .yml. The flow of the recognition phase starts by capturing live images of the classroom. These images are normalised and tested to detect human faces by employing Haar Cascade algorithm. After the detection process, the detected faces are compared with the prevailing trainer file. This is utilised to complete the recognition process. The comparison of images is implemented with the use the LBPH algorithm. As the name reveals, it compares histograms of the images and the recognised faces are identified with its reference ID. The attendance is marked present for the students whose faces are detected and this is stored in a separate attendance database.

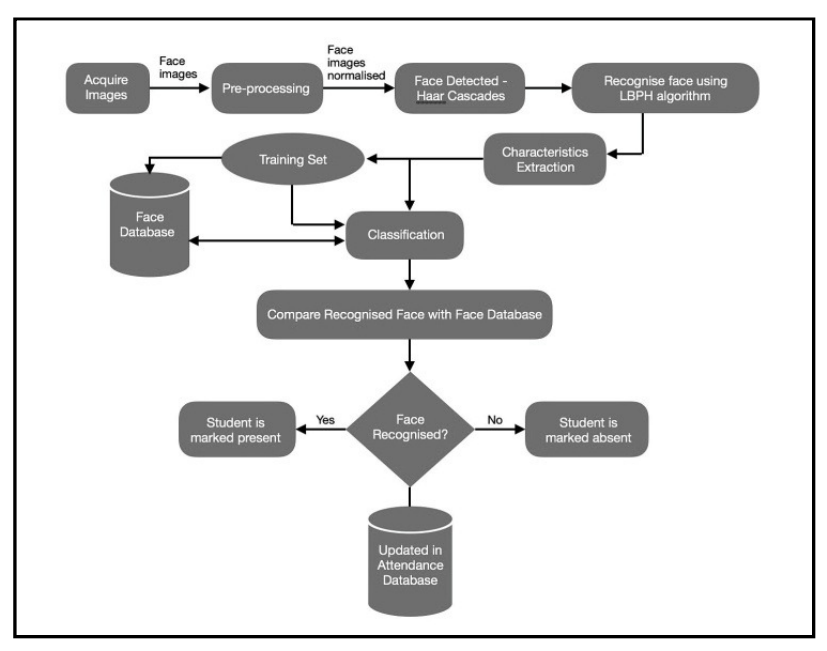

Figure 5: Block Diagram of the System

\section{EXPERIMENT RESULT AND DISCUSSIONS}

In this experimental design, students whose faces are individually captured achieve the best recognition level. Sometimes, during the recognition of faces, a student may be incorrectly recognised as another student. This can result in an incorrect acquisition of data. By considering the experimental results, further research should be associated with better techniques of feature extraction and recognition processes.

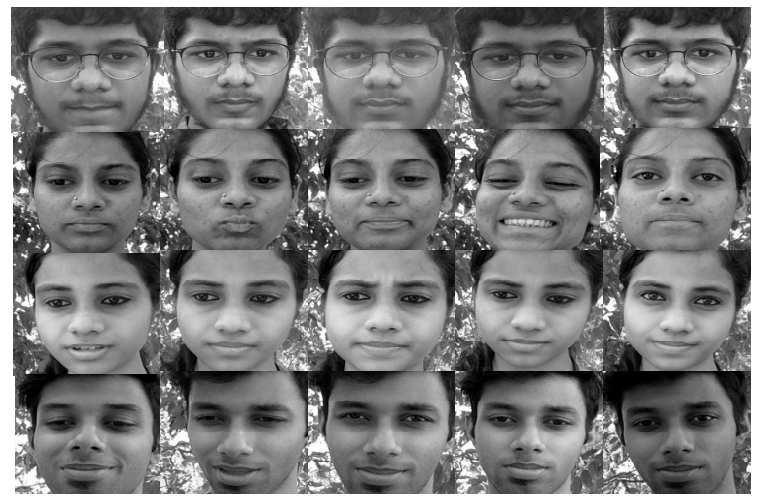

Figure 6: Five example facial training images for each

\section{VI.CONCLUSION}

In this paper, a system is put in place to automatically compute attendance of students using Facial recognition. It is implemented on a personal computer. The program is written using the Python programming language and the algorithms are implemented through the OpenCV library. It can be concluded that Automated Attendance System Using Facial Recognition implemented in the classroom works effectively. Certainly, it can be improved by enhancing the algorithms 
International Journal of Engineering and Techniques - Volume 4 Issue 2, Mar-Apr 2018

used for feature extraction and recognition processes. This can lead to a more efficient system for attendance. However, the current algorithms used are one of the best approaches and the performance of the system fulfils the aim of the paper.

\section{ACKNOWLEDGEMENT}

Nobody has been more important to us in the pursuit of this project than the members of our families. We would like to thank our parents, whose love and guidance are with us in whatever we pursue. Most importantly, we wish to thank our friends and professors who motivated us to complete this project in time.

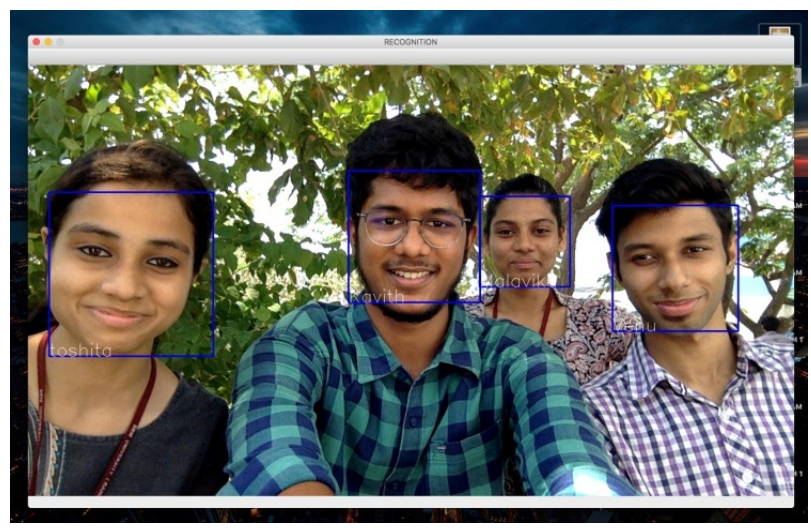

Figure 7: The face recognition system working successfully

\section{REFERENCES}

1. LBPH OpenCV: https://docs.opencv.org/2.4/modules/contrib/doc/facerec/facerec_tutorial.h tml\#local-binary-patterns-histograms

2. Local Binary Patterns: http://www.scholarpedia.org/article/Local_Binary_Patterns

3. http://citeseerx.ist.psu.edu/viewdoc/download?doi=10.1.1.417.2254\&rep= rep1\&type $=$ pdf

4. "Facial recognition system for automatic presence control in a classroom". IEEE Transaction on Information system and technology(2017);João Roberto de Toledo Quadros, Fabio Paschoal e Laercio Brito Gonçalves.

5. "Face recognition based on LBPH and regression of Local Binary features". IEEE Transaction on image processing(2017);Gao Xiang, Zhu Qiuyu, Wang Hui.

6. "Facial parts detection using Viola Jones algorithm". IEEE Transaction on Advanced computing and communication system(2017);K.Vikram, S. Padmavathi

7. "Student Attendance System in Classroom Using Face Recognition Technique"\%. IEEE Transaction on Information system(2017);Samuel Lukas, Aditya Rama Mitra, Ririn Ikana Desanti, Dion Krisnadi

8. https://thecodacus.com/face-recognition-loadingrecognizer/\#.WsW10GaB1QJ. 